'Sección Hematología, Hospital del Salvador. Santiago, Chile. ${ }^{2}$ Encargada ministerial protocolo Gammapatías monoclonales. Recibido el 12 de octubre de 2018, aceptado el 25 de mayo de 2019.

Correspondencia a: Camila Peña Avenida Salvador 364 Providencia, Santiago, Chile. camipena@gmail.com

\section{Paraproteínas: claridad en la nebulosa}

\author{
CAMILA PEÑA ${ }^{1,2}$
}

\section{Paraproteins. A review}

Hematological neoplasms are tumors of cells in different states of maturation and differentiation. Since monoclonal gammopathies $(M G)$ refer to $B$ mature lymphocyte neoplasms, lymphogenesis should be well known. We must keep in mind that the last stage of maturation of these lymphocytes is the plasma cell. This is how a MG could appear in the context of a plasma cell neoplasm, such as multiple myeloma or amyloidosis, but also in relation to a lymphoma. A monoclonal peak is produced by mature B lymphocytes or plasma cells that secrete a monoclonal protein (Immunoglobulin), and represents a MG. But it must be emphasized that, in the correct clinical context, a hypogammaglobulinemia can represent a $M G$ as well. Another important point is the understanding and interpretation of requested tests, such as protein electrophoresis (PEP), immunofixation (IFX) or serum free light chains (sFLC). The current MG screening panel includes these three studies (PEF, IFx, sFLC), although a simpler panel measuring PEF and sFLC has also been proposed, but not yet formally validated. Therefore, screening done only with PEP is insufficient.

(Rev Med Chile 2019; 147: 1036-1041)

Key words: Blood Protein Electrophoresis; Paraproteinemias; Paraproteins.
L as gammapatías monoclonales (GM) son entidades en general poco comprendidas para el internista, es por esta razón que esta revisión intentará dar una mirada diferente para el enfrentamiento diagnóstico de estas patologías, para que dejen de ser esa nebulosa en que a veces se convierten.

Primero algunas definiciones o conceptos:

Se define GM como un grupo de enfermedades caracterizadas por la proliferación clonal de linfocitos B maduros o de células plasmáticas, que producen un único tipo de cadena liviana y/o pesada (llamado componente monoclonal, componente $\mathrm{M}$, proteína $\mathrm{M}$ o paraproteína).

La monoclonalidad indica que estas células neoplásicas producen un único tipo de cadena pesada y/o liviana, por ejemplo, IgG Kappa, sólo cadenas livianas lambda, etc. Aquí cabe destacar que en muchos casos el resto de las inmunoglobulinas (Ig) (las no involucradas en la monoclonalidad o Ig policlonales) están disminuidas, a lo que se llama inmunoparesia.

\section{¿Cómo comprender las neoplasias hematológicas?}

Las neoplasias hematológicas en general son mejor comprendidas cuando se entiende su origen. Son neoplasias de células, no de "lugares". Es así como las leucemias agudas son neoplasias de células inmaduras, no "cáncer de médula ósea", o como los linfomas son neoplasias de linfocitos maduros, no "cáncer de ganglio". Este enfrentamiento de cualquier neoplasia hematológica llevará a una comprensión más acabada de la patología y sus alcances. La clasificación de la Organización Mundial de la Salud (WHO, por su sigla en inglés) clasifica de esta forma las neoplasias, siendo ésta la clasificación más didáctica ${ }^{1}$.

\section{Neoplasias de células plasmáticas (NCP)}

Es importante comprender que NCP no es sinónimo de mieloma múltiple, sino que hay una amplia gama de estas patologías, cada una con diferente presentación, grado de infiltración de 
médula ósea (MO) u otros órganos, evolución, etc.

Las NCP comprenden: la gammapatía monoclonal de significado incierto no IgM (GMSI no IgM), la gammapatía monoclonal de significado clínico, la gammapatía monoclonal de significado renal (GMSR), mieloma múltiple (MM) tanto asintomático o smoldering como activo, síndrome de POEMS, plasmocitomas (óseo y extraóseo/solitario o múltiple), leucemia de células plasmáticas y enfermedad por depósito de inmunoglobulina (Ig) monoclonales como la amiloidosis primaria (AL) o la enfermedad por depósito de cadenas pesadas o livianas.

Por otro lado, las NCP no son sinónimo de GM. Esta última se refiere solamente a la detección de una paraproteína en exámenes de laboratorio. Más específicamente, las NCP son parte de las GM.

\section{Ontogenia de la célula plasmática}

La célula plasmática es la fase terminal de un linfocito B. El linfocito B, como todas las células hematológicas, nace desde una stem cell o célula madre en la médula ósea (MO), que luego se diferencia a célula inmadura o linfoblasto B. Alteraciones a este nivel dan por resultado las patologías llamadas neoplasias de linfocitos B inmaduros o leucemias agudas linfoblásticas B. Todavía en la MO, el linfoblasto madura hacia un linfocito B maduro llamado "naive", ya que aún no ha tenido contacto con un antígeno. Es en esta fase se produce la "Recombinación V(D)J", que no es más que la expresión de inmunoglobulinas en la superficie de estas células, constituyendo el receptor antigénico. Es decir, desde este momento ya los linfocitos B tienen Ig, de las clases IgM e IgD.

Posteriormente, migran hacia órganos linfáticos, principalmente ganglios, pero también a otros como el bazo. Es ahí donde tienen contacto con el antígeno y ocurre la "hipermutación somática", que es la etapa en que la Ig se hace específica para su antígeno, seguido del "switch class o cambio de isotipo", en donde la Ig cambia de clase según la necesidad, llevando en esta fase al linfocito $B$ maduro poseer IgG (la más frecuente), IgA, IgM, $\operatorname{IgD}$ o IgE.

Luego, los linfocitos B entran a su fase más madura, transformándose en linfocitos $\mathrm{B}$ de memoria o células plasmáticas. Estas células plasmáticas migran nuevamente a la $\mathrm{MO}^{2}$. Cualquier alteración en estas fases (recombinación V(D)J, hipermutación somática o cambio de isotipo) lleva a neoplasia de linfocitos B maduros (linfomas y/o neoplasias de células plasmáticas). Esto se explica ya que es en estas fases donde puede haber inestabilidad y alteración del ADN celular.

Es por esto que en la clasificación de la WHO, las NCP se encuentran dentro de las "neoplasias de linfocitos B maduros" ${ }^{\text {. }}$

\section{Estudio de la paraproteína}

\section{Electroforesis de proteinas}

La electroforesis de proteínas (EFP) es un examen que separa las proteínas del suero (u orina) según carga eléctrica. Las proteínas del suero siempre "corren" de la misma forma, formando 5 regiones bien definidas. La primera y la más abundante es la albúmina. Hay que recordar que esta proteína da cuenta de $50 \%$ de las proteínas del suero. Luego se encuentra el grupo de las alfa, 1 y 2, cada una con sus proteínas características: $\alpha 1$-antitripsina, la $\alpha 1$-glucoproteína, la transcortina, la HDL y la $\alpha$-fetoproteína; Y la $\alpha 2$-macroglobulina, la haptoglobina y la ceruloplasmina. Más adelante está el grupo de las beta, en el que se encuentran la transferrina, la hemopexina, C3 del complemento y la $\beta$-lipoproteína o LDL. En esta región puede observarse además la Ig IgA cuando está elevada. Y es importante recordar que es no es infrecuente que el aumento del resto de las proteínas de esta región enmascaren estos peaks IgA, siendo a veces difícil de interpretar. Finalmente tenemos la zona gamma, donde se encuentran el resto de las Ig: G, M, D y E, siendo la IgG la más representada ${ }^{3}$.

La EFP es el método que cuantifica la paraproteína. Es así como las definiciones de MGUS y MM asintomático o smoldering, se basan en esta medición. Por lo tanto, la EFP cuantifica la paraproteína, pero no lo caracteriza.

\section{Interpretación}

Como ya se mencionó, la proteína $\mathrm{M}$ puede evidenciarse en la región beta, betagamma o gamma. Esto se observa como la clásica imagen de un peak delgado en una zona específica de gamma o beta.

Otra forma en que puede manifestarse una proteína $\mathrm{M}$ en la EFP es la hipogammaglobulinemia, que puede observarse en las GM de cadenas 
livianas (CL). Como su nombre lo indica, estas no tienen una sobreexpresión de ninguna Ig de cadena pesada, y por lo tanto, suelen presentarse como hipogammaglobulinemia (aplanamiento de la curva en gamma). En casos en que la CL involucrada sea de gran cuantía, este tipo de GM puede manifestarse con un pequeño peak en la EFP.

Dado que existen pocas causas de hipogammaglobulinemia, (inmunodeficiencias, posterior a fármacos como rituximab), este hallazgo en la EFP sumado a una clínica compatible, debe hacer sospechar en una GM como la primera causa. Es decir, un paciente que presenta hipogammaglobulinemia y alta sospecha clínica, obliga a buscar la paraproteína con técnicas más sensibles ${ }^{4}$.

Por otro lado, en la hipergammaglobulinemia difusa, a diferencia del peak monoclonal, se observa un aumento homogéneo y difuso de la curva en la zona gamma. Esto se observa generalmente en patologías inflamatorias, autoinmunes o infecciones crónicas. Su significado es el aumento de todas las Ig, es decir, un aumento de Ig policonal. En estos casos, los valores del recuento o cuantificación de Ig suelen estar por sobre los valores normales, sin embargo, este hecho no implica monoclonalidad.

\section{Electroforesis de proteínas en orina}

La técnica en orina es la misma que en suero, por lo que las proteínas encontradas se presentan en las mismas zonas previamente descritas. Sin embargo, en la orina normalmente no hay proteínas o sólo una mínima cantidad, por lo que las curvas normales de la EFP se ven planas, o con un pequeño aumento en la región de la albúmina.

En un síndrome nefrótico habrá mayor can-

Tabla 1. Causas posibles cuando se detecta una paraproteína

\begin{tabular}{|c|c|}
\hline \multicolumn{2}{|c|}{ Tiene un peak monoclonal... ¿qué es? } \\
\hline Neoplasias células plasmáticas & Neoplasia de linfocitos B maduros \\
\hline - Gamapatía monoclonal de significado incierto no lgM (GMSI no lgM) & - Macroglobulinemia de Waldenström \\
\hline - Gamapatía monoclonal de significado renal (GMSR) & - GMSI lgM \\
\hline - Mieloma múltiple (MM) (smoldering y sintomático) & - Linfoma linfoplasmocítico \\
\hline - POEMS & - LLC \\
\hline - Plasmocitoma (óseo y extraóseo/solitario o múltiple) & - Linfoma marginal esplénico \\
\hline - Leucemia de células plasmáticas & - MALT \\
\hline - Amiloidosis primaria (Al) & - Linfoma folicular \\
\hline & - DCGB \\
\hline
\end{tabular}

\section{Diferenciación LB a célula plasmática}

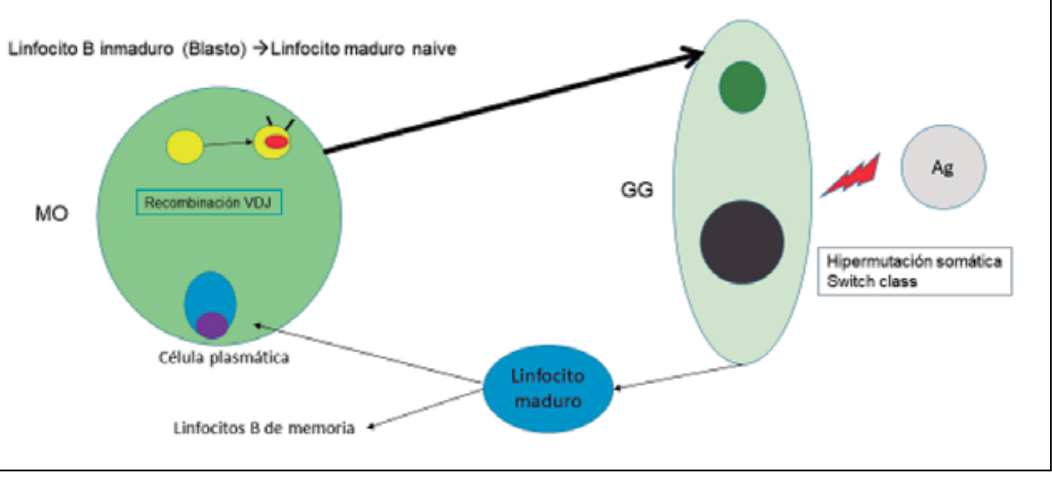

Figura 1. Esquema de la maduración del linfocito $B(L B)$. MO: médula ósea. GG: ganglio. Ag: antígeno. 
tidad de albúmina, por lo que habrá una mayor curva en esa zona. En alteraciones glomerulares, habrá de todo tipo de proteínas. En una amiloidosis, en general se ve un aumento en la albúmina y un peak más bien pequeño en la zona gamma. En un riñón de MM también se ve aumento de albúmina y un peak mayor en gamma.

\section{Inmunofijación}

La inmunofijación (Ifx) es un examen que puede realizarse también en suero u orina. Este examen caracteriza la paraproteína, no lo cuantifica como lo hace la EFP. Es por esto que ambos son exámenes que se complementan, no se excluyen uno al otro.

Esta se caracteriza por la realización de 6 EFP en tándem del mismo paciente. Luego se suministra un "anticuerpo anti anticuerpo", es decir, suero anti IgA (cadena pesada alfa), anti IgG (cadena pesada gama), anti IgM (cadena pesada mu), Anti Kappa y anti Lambda. Luego se "lavan" estos anticuerpos y se observa la precipitación obtenida, y si ésta es difusa u homogénea ${ }^{5}$. Se habla de monoclonalidad si existe precipitación homogénea.

Es importante tener en cuenta que por la orina generalmente lo que filtra son las CL, no la Ig pesada, por lo que no es extraño observar por ejemplo en la Ifx en suero una paraproteína "IgG Kappa" y en la orina sólo "Kappa".

Otro aspecto que se debe recordar, es que no se realizan de rutina la IgD e IgE por inmunofijación, por lo que si se sospecha este tipo de paraproteína debe solicitarse activamente.

\section{Recuento o cuantificación de inmunoglobulinas}

Tal como su nombre lo indica, este examen cuantifica las Igs. En general se realiza recuento de la IgA, IgM, e IgG. Es importante recalcar que no mide clonalidad, mide la concentración total de cada Ig. Es decir, la cuantificación de IgG mide tanto la IgG Kappa + IgG Lambda. Esto se puede ejemplificar en que una cuantificación de IgG normal no descarta monoclonalidad, y por el otro lado, una IgG aumentada puede observarse en una hipergammaglobulinemia policlonal. Debe recordarse que estos exámenes deben interpretarse siempre en conjunto.

Aquí es importante también conocer el concepto de inmunoparesia. Esta se refiere a la disminución de las Ig no involucradas. Por ejemplo, si la paraproteína es IgG, se observa disminución de
IgM e IgA. De hecho, se observa inmunoparesia en hasta $90 \%$ de los $\mathrm{MM}^{6}$.

Por otro lado, debe recordarse que las IgE e IgD no se cuantifican de rutina. Como ya se mencionó anteriormente, al encontrarse un peak monoclonal pequeño con el recuento de Ig disminuidos, lo más probable es una GM de CL, pero siempre debe sospecharse además que podría tratarse de una paraproteína IgE e IgD, las que deben solicitarse activamente.

\section{Cadenas livianas libres en suero}

Este examen es el más reciente y añadió sensibilidad al estudio de las $\mathrm{GM}^{7}$. Se estudia por nefelometría y es también un anticuerpo, que en este caso se une a una región "escondida" de las $\mathrm{CL}$, que sólo se "muestra" al estar la cadena liviana libre (CLL). De allí su nombre. Es un examen que cuantifica, tanto a la CL lambda (L) como a la CL kappa (K) libre. No mide clonalidad, sino que ésta se infiere mediante la razón $\mathrm{K} / \mathrm{L}^{8}$.

Su mayor utilidad, como ya se mencionó, es agregar sensibilidad al panel de tamizaje de las GM. En cuanto a tipificación de la GM, debe recordarse que el MM de cadenas intactas tiene una fracción de CLL, hasta en $96 \%$ de los casos ${ }^{9}$, por lo tanto es útil en todos los tipos de MM. Destaca dentro de este grupo los MM llamados "no secretores" (antiguamente definidos como MM con MO infiltrada, clínica compatible, pero EFP e IFx negativas), en los cuales se ha detectado con este examen secreción de CLL hasta 70\% de los casos. Estos son los llamados "MM oligosecretores" actualmente.

\section{¿Qué patologías pueden presentarse con una paraproteína?}

Siempre se enseña que un peak monoclonal es sinónimo de MM, lo que dista de la realidad. Para comprender esto, se debe recordar la ontogenia del linfocito B previamente explicado. El linfocito B (LB) puede secretar Ig desde su fase de LB maduro "naive" en adelante. Es decir, cualquier neoplasia de linfocitos B maduros es capaz de secretar proteína $M$, ya sea neoplasia de células plasmática o cualquier linfoma. Previo al cambio de isotipo (switch class) o hipermutación somática, generalmente la Ig secretada es IgM o menos frecuentemente IgD. Es por esta razón que si la 
paraproteína encontrada es IgM, es más probable un síndrome linfoproliferativo que una neoplasia de células plasmáticas. Entre las GM IgM la más frecuente es la Macroglobulinemia de Waldenstrom, que es un tipo de linfoma. Sin embargo, una paraproteína IgM puede encontrarse también en el linfoma MALT, la leucemia linfática crónica/linfoma de linfocitos pequeños, el linfoma folicular, o incluso el linfoma difuso de células grandes B.

Y por el contrario, si la paraproteína es no-IgM, lo más probable es que se trate de una NCP.

\section{Panel de screening (tamizaje) en GM}

Ante la sospecha de una GM se suele solicitar sólo una EFP en suero, lo que es un error. La EFP por sí sola tiene una sensibilidad de $89 \%$ en los MM de cadena intacta, $40-50 \%$ en los MM de CL, 50-60\% en amiloidosis AL, 72\% en los plasmocitomas óseos, $10 \%$ plasmocitomas extraóseos, y $0 \%$ de los MM no secretores. Es decir, muchas GM simplemente no se diagnosticarán. Y aún peor, al no saber interpretar este examen, muchas veces se descarta el diagnóstico de GM por encontrarse una hipogammaglobulinemia (y no un peak), lo que como se explicó previamente es erróneo.

El panel de tamizaje en sospecha de GM recomendado por los expertos es EFP en suero, Ifx en suero y CLL en suero. La sensibilidad en el diagnóstico de MM de cadena intacta con EFP e Ifx sube a 93\%, y sumado a CLL a 97\%. Este panel se realiza sólo en suero, y tiene por tanto la ventaja de prescindir de exámenes de orina, siempre laboriosos y engorrosos. La única patología que aún requiere estudio de orina para su tamizaje es la amiloidosis $\mathrm{AL}^{10-15}$.

Un panel más sencillo se ha propuesto como tamizaje actualmente, aunque no ha sido validado. Este es EFP en suero + CLL en suero, panel sencillo $\mathrm{y}$ asequible ${ }^{16,17}$.

\section{Puntos a destacar}

Las NCP son parte de las GM, no sinónimo.

Las neoplasias hematológicas son neoplasias de células. En el caso de las GM es vital conocer y entender la ontogenia del linfocito B.

La sospecha de MM o cualquier GM no sólo se da con la presencia de un peak monoclonal en la EFP. También es importante sospecharlo con una hipogammaglobulinemia.
La hipergammaglobulinemia difusa es policlonal.

Una paraproteína puede secretarse por un linfocito B maduro o célula plasmática, es decir, debe pensarse en una NCP o en un linfoma, según la clínica de presentación.

El tamizaje ante la sospecha de una GM es con EFP, Ifxs y CLL en suero. No es correcto solicitar sólo EFP.

Debe solicitarse exámenes en orina si se sospecha amiloidosis AL.

\section{Referencias}

1. McKenna RW, Kyle RA, Kuehl WM, Harris NL, Coupland RW, Fend F. Plasma Cell neoplasms. In: Swerdlow SH; Campo E; Lee Harris N; Jaffe ES; Pileri SA; Stein H; Thiele J. WHO Classification of tumors of Haematopoietic and Lymphoid Tissues: 239-56. Revised $4^{\text {th }}$ Ed. International Agency for Research on Cancer, 2016. Lyon. France.

2. Corre J, Munshi N, Avet-Loiseau H. Genetics of multiple myeloma: another heterogeneity level? Blood 2015; 125 (12): 1870-6.

3. O'Connell TX, Horita TJ, Kasravi B. Understanding and interpreting serum protein electrophoresis. Am Fam Physician 2005; 71 (1): 105-12.

4. Keren DF, Schroeder L. Challenges of measuring monoclonal proteins in serum. Clin Chem Lab Med 2016; 54 (6): 947-61.

5. Attaelmannan M, Levinson SS. Understanding and identifying monoclonal gammopathies. Clin Chem 2000; 46 (8 Pt 2): 1230-8.

6. Sørrig R, Klausen TW, Salomo M, Vangsted AJ, Frølund UC, Andersen KT, et al. Immunoparesis in newly diagnosed Multiple Myeloma patients. PLoS One 2017; 12 (12): e0188988.

7. Bradwell AR, Carr-Smith HD, Mead GP, Tang LX, Showell PJ, Drayson MT, et al. Highly sensitive, automated immunoassay for immunoglobulin free light chains in serum and urine. Clin Chem 2001; 47: 673-80.

8. Dispenzieri A, Kyle R, Merlini G, Miguel JS, Ludwig H, Hajek R, et al. International Myeloma Working Group guidelines for serum-free light chain analysis in multiple myeloma and related disorders. Leukemia 2009; 23 (2): 215-24.

9. Durie BG, Harousseau JL, Miguel JS, Blade' J, Barlogie $\mathrm{B}$, Anderson $\mathrm{K}$, et al. International uniform response criteria for multiple myeloma. Leukemia 2006; 20: 146773. 
10. Kang SY, Suh JT, Lee HJ, Yoon HJ, Lee WI. Clinical usefulness of free light chain concentration as a tumor marker in multiple myeloma. Ann Hematol 2005; 84: 588-93.

11. Katzmann JA, Dispenzieri A, Kyle RA, Snyder MR, Plevak MF, Larson DR, et al. Elimination of the need for urine studies in the screening algorithm for monoclonal gammopathies by using serum immunofixation and free light chain assays. Mayo Clin Proc 2006; 81: 1575-8.

12. Hill PG, Forsyth JM, Rai B, Mayne S. Serum free light chains: an alternative to the urine Bence Jones proteins screening test for monoclonal gammopathies. Clin Chem 2006; 52: 1743-8.

13. Abadie JM, Bankson DD. Assessment of serum free light chain assays for plasma cell disorder screening in a veterans affairs population. Ann Clin Lab Sci 2006; 36: 157-62.
14. Bakshi NA, Gulbranson R, Garstka D, Bradwell AR, Keren DF. Serum free light chain (FLC) measurement can aid capillary zone electrophoresis in detecting subtle FLC-producing M proteins. Am J Clin Pathol 2005; 124 : 214-8.

15. Marien G, Oris E, Bradwell AR, Blanckaert N, Bossuyt $\mathrm{X}$. Detection of monoclonal proteins in sera by capillary zone electrophoresis and free light chain measurements. Clin Chem 2002; 48: 1600-1.

16. Katzmann JA, Kyle RA, Benson J, Larson DR, Snyder MR, Lust JA, et al. Screening Panels for Detection of Monoclonal Gammopathies. Clin Chem 2009; 55 (8): 1517-22.

17. Peña C, Ortiz M, Voisin J, Peralta A, Balboa V, Delgado F. Diagnostic accuracy of serum protein electrophoresis and free light chain measurements for monoclonal gammopathies. Rev Med Chile 2018; 146 (1): 64-7. 